\title{
Monochromated TEM/STEM and High-Resolution Electron Energy- Loss Spectroscopy (HREELS) of Extraterrestrial Materials
}

\author{
Z. R. Dai, ${ }^{1}$ J. P. Bradley, ${ }^{1}$ G. Graham, ${ }^{1}$ R. Erni, ${ }^{2}$ and N. Browning ${ }^{2,3}$ \\ ${ }^{1}$ Institute of Geophysics and Planetary Physics, Lawrence Livermore National Laboratory, \\ Livermore, CA 94551 \\ ${ }^{2}$ Dept. of Chemical Engineering and Materials Science, UC Davis, Davis, CA 95616 \\ ${ }^{3}$ National Center for Electron Microscopy, Lawrence Berkeley National Laboratory, \\ Berkeley, CA 94551
}

A NASA-funded $200 \mathrm{keV}$ FEI Tecnai F20 G ${ }^{2}$ XT monochromated (scanning) transmission electron microscope has recently been installed at Lawrence Livermore National Laboratory. The microscope is equipped with a voltage-stabilized high tension (HT) supply, monochromator, high-resolution electron energy-loss spectrometer/energy filter, high-resolution annular dark-field detector, and a solid-state X-ray energydispersive spectrometer. The high-tension tank has sections for $60 \mathrm{~Hz}$ and high frequency filtering to provide an operating voltage of $200 \mathrm{kV} \pm 0.005 \mathrm{~V}$. The energy filter is a Gatan model 866 Tridiem-ERS high-resolution GIF capable of $\leq 0.15 \mathrm{eV}$ energy resolution. The X-ray detector (EDAX/Genesis 4000) has a take-off angle of $20^{\circ}$, an active area of 30 $\mathrm{mm}^{2}$, and a solid angle of 0.3 steradians.

The monochromator and GIF provide high-resolution electron energy-loss spectroscopy (HREELS) energy resolution similar to synchrotron XANES, but with $~ 100 \mathrm{X}$ better spatial resolution. The low-loss or valence electron energy-loss spectroscopy (VEELS) region $(0-100 \mathrm{eV})$ includes the visible (VIS) and ultraviolet (UV) absorption regions. We are particularly interested in this region because we are want to directly compare the optical properties of extraterrestrial grains in the electron microscope with those of dust grains in space. Most of the VEELS features we are detecting are being observed for the first time in extraterrestrial materials. Figure 1 compares HREELS spectra from a fluid inclusion within glass with the surrounding glass matrix within an interplanetary dust particle (IDP). The fluid inclusion spectrum (red) has at least ten edges (labeled a-j) superimposed on the volume plasmon. Edges b, c, e \& $\mathrm{g}$ are due to $\mathrm{SiO}_{4}{ }^{4-}$ excitons, $\mathrm{h}$ is a surface plasmon and $\mathrm{k}$ is the energy maximum of the volume plasmon. Edges $\mathrm{a}, \mathrm{d}, \mathrm{f}, \mathrm{i}$ and $\mathrm{j}$ are specific to the fluid inclusion. "a" also correlates with a pre-edge on the oxygen$\mathrm{K}$ feature at $\sim 532 \mathrm{eV}$, suggesting that "a" may be due to $\mathrm{O}_{2}$ [1]. The $\mathrm{i}$ and $\mathrm{j}$ edges from the fluid inclusion may be related to "a", although the position of $\mathrm{j}(\sim 22 \mathrm{eV})$ is consistent with the Helium-K core excitation edge and some IDPs contain saturation doses of implanted solar wind Helium. Time-resolved in-situ observations indicate that the intensities of edges $a, d, f, i$, and $j$ decrease as the contents of the fluid inclusion are released as the glass degrades in the beam (Fig. 1(B)). Using VEELS we have also detected a $5.7 \mathrm{eV}(2175 \AA$ ) feature in IDPs (Fig. 1(C)). An astronomical extinction feature at $2175 \AA$ is by far the strongest UV-VIS spectral signature of interstellar dust observed by astronomers. The carrier grains are organic carbonaceous matter and GEMS, some with non-solar isotopic compositions confirming that they are interstellar grains [2]. 
We are using X-ray mapping and energy filtered imaging to provide information about the distribution of major elements at the nanometer scale in IDPs (Fig. 2). Energy filtered imaging is particularly suitable for high spatial resolution mapping of light elements $(\mathrm{C}$, $\mathrm{N}, \mathrm{O}$ ), as well as heavier elements like $\mathrm{Mg}, \mathrm{Si}, \mathrm{S}, \mathrm{Fe}, \mathrm{Ni}$, with acquisition times of 4-60 seconds and spatial resolution of 1-2 $\mathrm{nm}$. In addition, the distribution of valence states of some elements can be mapped. X-ray mapping is also capable of high spatial resolution but greatly extended acquisition times and high-brightness nanoprobes are required, placing significant constraints on the types of materials that can be mapped. High x-ray spatial resolution is typically achieved at the expense of good counting statistics.

\section{References}

[1] R. Erni et al., Micron (2005) (in press).

[2] J.P. Bradley et al., Science 307 (2005) 244.

[3] G. Graham et al., Lunar Planet. Sci. 35 (2004) 2044.
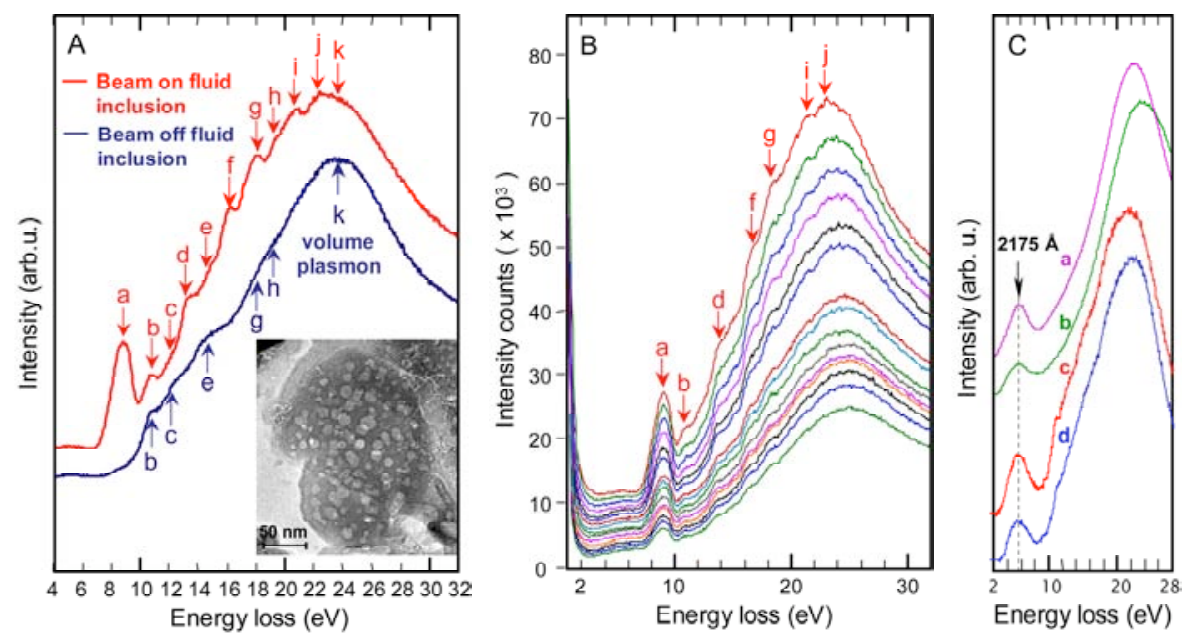

Fig. 1. (A) HREELS spectra from a $\sim 10 \mathrm{~nm}$ diameter fluid inclusion (red) within aluminosilicate glass in IDP W7013E-17 and glass matrix alongside fluid inclusion (blue). Inset is a TEM image of the grain. (B) Time-resolved HREELS spectra from a fluid inclusion in IDP (W7013E17). (C) VEELS spectra from IDPs showing a $5.7 \mathrm{eV}(2175 \AA)$ feature.
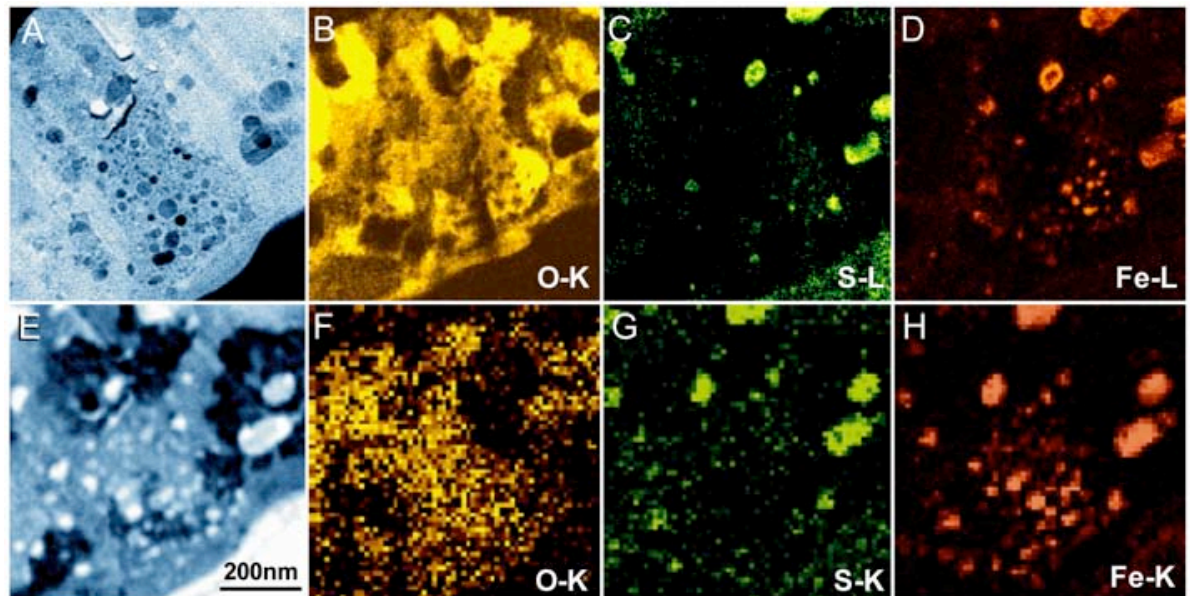

Fig. 2. Comparison of energy filtered TEM imaging (B, C \& D) and X-ray mapping $(F, G \& H)$ from IDP L2036-G16. TEM sample was prepared by FIB technique [3]. (A) is zero-loss filtered image, and (E) is corresponding annular dark-field STEM image. 